\title{
Curvature continuous bi-4 constructions for scaffold- and sphere-like surfaces
}

\author{
Kęstutis Karčiauskas ${ }^{a}$ and Jörg Peters ${ }^{b} \quad{ }^{a}$ Vilnius University ${ }^{b}$ University of Florida
}

\begin{abstract}
Scaffold surfaces bound geometric structures that have a dual characterization as a curve network and a solid. A subset of scaffold surfaces can be modeled with minimal single-valence (MSV) meshes, i.e. meshes consisting of vertices of a single irregular valence $n$ two of which are separated by exactly one regular, 4-valent vertex. We present an algorithm for constructing piecewise biquartic surfaces that join with curvature continuity to form scaffold surfaces for MSV meshes, for $n=5, \ldots, 10$. Additionally, for sphere-like meshes, we exhibit bi-quartic curvature continuous surfaces with polar parameterization.
\end{abstract}

Keywords: curvature continuity, scaffold surface, multi-sided, bi-4 spline, free-form surface

\section{Introduction}

Carbon nano structures, skeletonized solids, branching vascular trees of the circulatory system, novel weave-like 3D printed materials, scaffolds for regrowing body parts, certain self-supporting architectural or biomimetic structures share a dual characterization as curve networks and as solids. Often these structures are embedded into and interact with fields such as fluids, pressure from interspersed deformable materials, outside forces, etc. so that the smoothness of the surface of the solid representation as the interface plays an important role, e.g. to encourage or discourage attachment and growth of tissue on the skeleton solid. Curvature continuity of such scaffold surfaces is of interest for flow computations, for example when applying the 'finite volume' method. While one can model

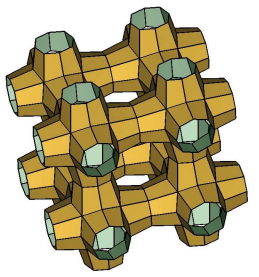

(a) MSV mesh

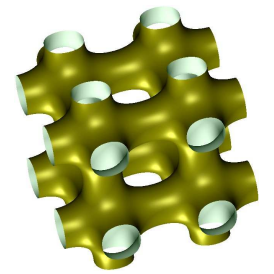

(b) curvature continuous scaffold surface

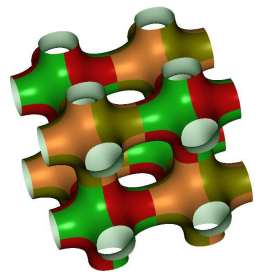

(c) neighborhoods of same valence
Figure 1: $\mathbf{G}^{\mathbf{2}} \mathbf{b i - 4}$ scaffold surface from MSV mesh with extraordinary nodes of valence 6 .

scaffold surfaces with general curvature continuous $\left(G^{2}\right)$ constructions, the similarity and potentially large number of scaffold branches (Fig. 1a) make it worthwhile to search for particularly simple constructions of branches, with good curvature distribution but fewer pieces and/or of lower polynomial degree and complexity than general $G^{2}$ constructions.

In this paper we derive such simple building blocks of curvature continuous free-form scaffold surfaces: the most basic scaffold surfaces arise from networks that admit quad-meshing with a single repeated valence $n$ other than valence 4 (since

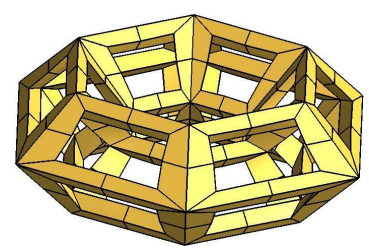

(a) MSV mesh

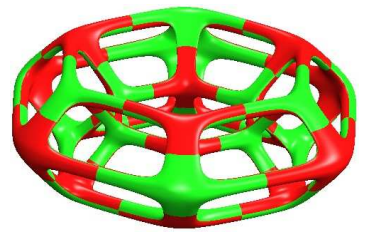

(b) scaffold surface
Figure 2: $\mathbf{G}^{\mathbf{2}}$ bi-4 surface of genus 25 from MSV mesh with extraordinary nodes of valence 8 .

$n=4$ corresponds to the tensor-product mesh). These are meshes assembled from pieces that have exactly $n$ quadrilaterals clustered around each $n$-valent point (Fig. 1a, Fig. 2a, see also Fig. 18 for a carbon nano structure modelled by scaffold surface.) Surprisingly such minimal single valence (MSV) surfaces admit $G^{2}$ constructions using $2 \times 2$ patches of degree bi- 4 (see Fig. 1c, Fig. 2b), or, alternatively, just one bi-5 patch per quad. Both options will be presented and complemented by a bi-4 construction for sphere-like surfaces.

The core technical achievement in deriving these 'minimal' scaffold surfaces is to explicitly resolve the tightly interdependent second-order smoothness constraints - whose complexity seems to preclude an explicit solution - and to harness the remaining degrees of freedom in such a way as to obtain a good distribution of curvature over a set of challenging MSV meshes.

Overview. After a brief review of the state-of-the-art of high-quality $G^{2}$ surface constructions, Section 2 defines MSV meshes and the notation. Section 3 derives a bi-5 construction and Section 4 derives an alternative bi- $4 G^{2}$ construction. Section 5 adds spherical surfaces of degree bi-4.

\subsection{Literature: curvature continuous surfaces}

Multi-sided blends naturally arise when designing surfaces even of simple topology but complex geometry. Early $G^{2}$ constructions for multi-sided blends [GH89, GZ99, Pet02a, Pra97, 


\begin{tabular}{cll} 
degree & $\begin{array}{l}\text { pieces } \\
\text { per quad }\end{array}$ & reference \\
\hline & & \\
bi-9 & 1 & {$[$ Ye97, Kic13] } \\
bi-7 & 1 & {$[$ LS08] } \\
bi-6 & 1 & {$[$ KP16] } \\
bi-5 & $2 \times 2$ & {$[$ KP15a] }
\end{tabular}

Table 1: Polynomial $G^{2}$ constructions (with good curvature distribution on challenging test data).

Rei98] focused on the then hard task of enforcing the formal mathematical constraints of curvature continuity. In the past decade the emphasis has shifted to achieving better highlight lines and distribution of curvature. Since a formal mathematical definition of good shape is illusive, highlight lines and curvature are tested on a representative set of challenging input meshes such as [KP]. A consensus exists that oscillations in highlight lines and curvature distribution are to be avoided.

Table 1 summarizes recent advances in modeling high quality curvature continuous surfaces of moderate polynomial degree for a given, unrestricted quad-mesh layout. Degree bi-6 for a single patch per quad and degree bi- 5 for $2 \times 2$ patches per quad are thought to be the minimal degree for obtaining good shape. By focusing on a special yet useful class of quad-meshes, the constructions in this paper further reduce the polynomial complexity of $G^{2}$ surfaces without sacrificing good shape.

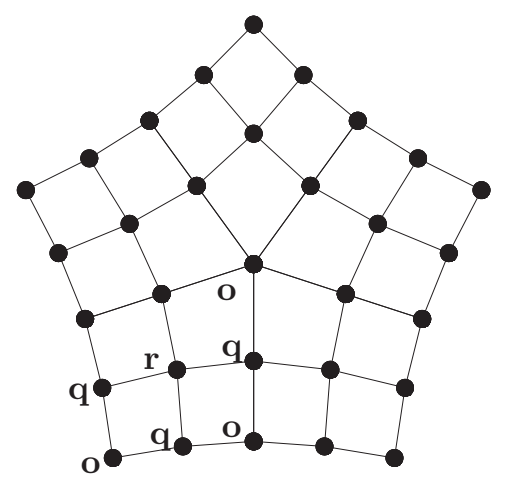

(a) o-centered view of input quad net $\mathbf{c}$

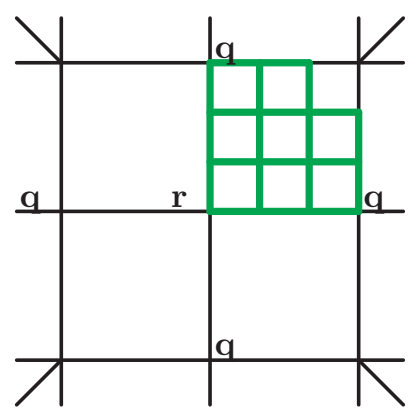

(c) r-centered view

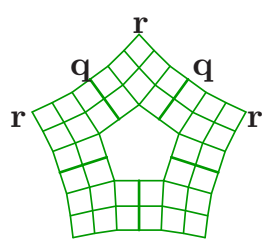

(b) tensor-border $\mathbf{b}$

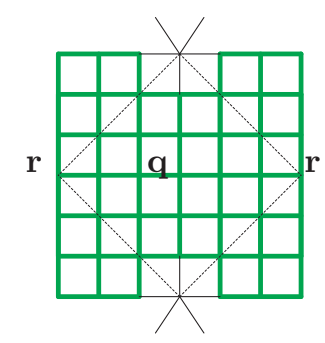

(d) q-centered view

Figure 3: MSV meshes. The unlabeled vertices of the same valence $n$ are of type o.

\section{MSV meshes and Setup}

In the following, we will focus on a special patchwork of quadrilateral facets (quads). As usual, nodes where four quads meet are called regular, while the remaining ones are called $e x$ traordinary nodes and are labeled o. For much of the exposition, we assume that all extraordinary nodes have the same valence $n>4$ (specifically $n \in\{5,6,7,8,9,10\}$ ) and each quad is uniquely associated with one extraordinary node that is one of its vertices. That is, extraordinary nodes o are separated by exactly one regular node of type $\mathbf{q}$ and four extraordinary nodes share one regular node of type $\mathbf{r}$ (see Fig. 3). A surface of genus $g$ constructed with such a quad mesh has $\frac{4(2-2 g)}{4-n}$ extraordinary nodes of valence $n$. We call this an MSV mesh (minimal single-valence mesh) - MSV meshes with $n=4$ are tensor-product meshes, and MSV meshes with $n=3$ form a partition of a cube and yield sphere-like meshes. Sphere-like surfaces are discussed at the end of the paper.

We construct tensor-product patches $\mathbf{f}$ of bi-degree $d$ in Bernstein-Bézier form (BB-form)

$$
\mathbf{f}(u, v):=\sum_{i=0}^{d} \sum_{j=0}^{d} \mathbf{f}_{i j} B_{i}^{d}(u) B_{j}^{d}(v), \quad u, v \in[0,1],
$$

where $B_{k}^{d}(t)$ are the Bernstein-Bézier (BB) polynomials of degree $d$ and $\mathbf{f}_{i j}$ are the BB-coefficients.

Interpreting the nodes of the MSV mesh as bi-cubic Bspline control points, B-spline to BB-form conversion (see e.g. [FAR02, PBP02]) is well-defined except for the BB-coefficients shared by $n \neq 4$ patches. The interpretation yields secondorder Hermite data along and across edges between the regular points $\mathbf{r}, \mathbf{q}$. Surrounding each $\mathbf{o}$, the Hermite data define a tensor-border $\mathbf{b}$ (of depth 2 and degree 3) in terms of BBcoefficients, see Fig. 3b.

Curvature continuity is verified by relating adjacent surface pieces via a reparameterization $\rho$ so that $\tilde{\mathbf{f}}=\mathbf{f} \circ \rho$.

Definition 1. Two surface pieces $\tilde{\mathbf{f}}$ and $\mathbf{f}$ sharing a boundary curve e join $G^{2}$ if there is a suitably oriented and non-singular reparameterization $\rho: \mathbb{R}^{2} \rightarrow \mathbb{R}^{2}$ so that the jets $\partial^{k} \tilde{\mathbf{f}}$ and $\partial^{k}(\mathbf{f} \circ$ $\rho), k=0,1,2$, agree along $\mathbf{e}$.

Throughout this paper e will correspond to patch parameters $(u, 0)$, i.e. $v=0$. For each e the chain rule of differentiation yields the well-known constraints (see e.g. [GH89, Pet02b])

$$
\begin{aligned}
& 0= \partial_{v} \tilde{\mathbf{f}}-a(u) \partial_{v} \mathbf{f}-b(u) \partial_{u} \mathbf{f}, \\
& \begin{aligned}
0= & \partial_{v}^{2} \tilde{\mathbf{f}}-a^{2}(u) \partial_{v}^{2} \mathbf{f}-2 a(u) b(u) \partial_{u} \mathbf{f} \partial_{v} \mathbf{f}-b^{2}(u) \partial_{u}^{2} \mathbf{f} \\
& \quad-e(u) \partial_{u} \mathbf{f}-d(u) \partial_{v} \mathbf{f}, \quad \text { where }
\end{aligned} \\
& \rho_{2}:=\left(u+b(u) v+\frac{1}{2} e(u) v^{2}, a(u) v+\frac{1}{2} d(u) v^{2}\right),
\end{aligned}
$$

is the Taylor expansion up to and including degree 2 of $\rho$ with respect to $v$.

\section{Bi-5 construction for MSV meshes}

In this section we build a cap of $n>4$ patches, one per quad, of degree bi-5 surrounding one extraordinary node o (Fig. 3). 


\subsection{Reparameterizing between sectors and solving constraints}

As shown in Fig. 4a, adjacent patches $\mathbf{p}^{k-1}$ and $\mathbf{p}^{k}, k=$ $0, \ldots, n-1$ (superscript modulo $n$ ) join along their shared boundary curve $\grave{\mathbf{p}}(u, 0)=\grave{\mathbf{p}}(u, 0)$, where (see Fig. $4 \mathrm{~b}$ ) $\grave{\mathbf{p}}:=$ $\mathbf{p}^{k-1}$ and $\mathbf{p}:=\mathbf{p}^{k}$. (When solving the constraint systems (1) and (2), it is convenient to index the BB-coefficients of adjacent patches $\mathbf{p}$ and $\mathbf{p}$ locally, symmetric with respect to the sector boundary curve rather than rotationally symmetric about the extraordinary point as is appropriate for the full surface cap in terms of $\mathbf{p}^{k}$.) To treat adjacent patches without bias for one side along the inter-sector boundary, in formula (3) we must set $a(u):=-1$ (so that $\partial_{v} \tilde{\mathbf{f}}$ and $\partial_{v} \mathbf{f}$ are weighted equally in (1)) and $e(u):=b(u)\left(b^{\prime}(u)-\frac{d(u)}{2}\right)$ (for similar symmetry in (2)). Our setup allows choosing

$$
b(u):=2 \mathrm{c}(1-u), d(u):=0, \text { where } c:=\cos \frac{2 \pi}{n} .
$$

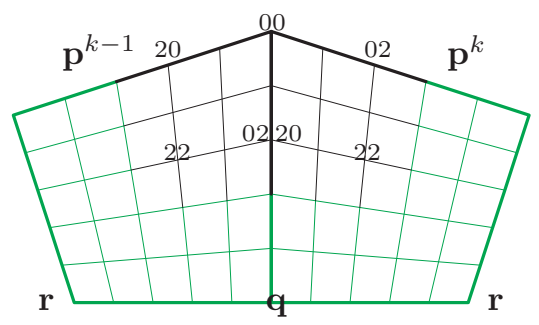

(a) rotationally-symmetric indexing

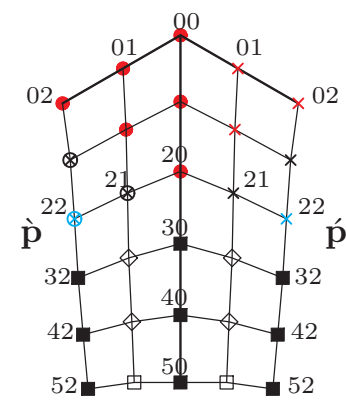

(b) sector-symmetric indexing

Figure 4: Indexing: In both indexing systems the extraordinary point has index 00 and the junction of boundary curves of tensor-border $\mathbf{b}$ of adjacent sectors (labeled $k-1$ respectively $k$ ) is denoted by 50 . (a) The reparameterized input tensor-border $\mathbf{b}$ is rendered in green. (b) The locally independent (free) BBcoefficients are marked by black and red disks, black and cyan circled crosses and the black squares.

The $G^{1}$ and $G^{2}$ constraints (1) and (2) between two patches then amount to setting to zero two polynomials of degree 5 . This yields $n$ local systems between sectors $k-1$ and $k$ for a total of 12 linear equations in the BB-coefficients. We solve these 12 equations symbolically for a subset of the BB-coefficients and optimize shape with the remaining coefficients. The explicit symbolic solutions in the remaining free coefficients are as follows.

(S1) We account for the interactions between the $n$ local systems of equations at the extraordinary point $\grave{\mathbf{p}}_{00}$ by selecting the six BB-coefficients $\mathbf{p}_{i j}^{0}, 0 \leq i+j \leq 2$, that define a quadratic expansion at extraordinary point of the sector with index $k=0$ and then express the corresponding BB-coefficients of other sectors recursively via

$$
\begin{aligned}
\dot{\mathbf{p}}_{00}:=\grave{\mathbf{p}}_{00}, \quad \dot{\mathbf{p}}_{10}:=\grave{\mathbf{p}}_{10}, \quad \dot{\mathbf{p}}_{20}:=\grave{\mathbf{p}}_{20} \\
\dot{\mathbf{p}}_{01}:=-\grave{\mathbf{p}}_{01}+2 c \grave{\mathbf{p}}_{10}+2(1-\mathrm{c}) \grave{\mathbf{p}}_{00} ; \\
\dot{\mathbf{p}}_{11}:=-\grave{\mathbf{p}}_{11}+\left(2-\frac{8 c}{5}\right) \grave{\mathbf{p}}_{10}+\frac{8 \mathrm{c}}{5} \grave{\mathbf{p}}_{20} ; \\
\dot{\mathbf{p}}_{02}:=\grave{\mathbf{p}}_{02}-5 c \grave{\mathbf{p}}_{11}+4 c^{2} \grave{\mathbf{p}}_{20}+(5 \mathrm{c}-4) \grave{\mathbf{p}}_{01} \\
+(1-\mathrm{c})\left(9 \mathrm{c} \grave{\mathbf{p}}_{10}+(4-5 \mathrm{c}) \grave{\mathbf{p}}_{00}\right) .
\end{aligned}
$$

The last three assignments in (5) correspond to the red crosses in Fig. 4b.

(S2) The assignments in each local system of black crosses

$$
\begin{aligned}
\dot{\mathbf{p}}_{21}:=-\grave{\mathbf{p}}_{21}+\left(2-\frac{6 c}{5}\right) \grave{\mathbf{p}}_{20}+\frac{6 c}{5} \grave{\mathbf{p}}_{30} \\
\dot{\mathbf{p}}_{12}:=\grave{\mathbf{p}}_{12}+\frac{4(5-4 c)(1-\mathrm{c})}{5} \grave{\mathbf{p}}_{10}+4(\mathrm{c}-1) \grave{\mathbf{p}}_{11} \\
+\frac{4 \mathrm{c}(9-7 \mathrm{c})}{5} \grave{\mathbf{p}}_{20}-4 \mathrm{c} \grave{\mathbf{p}}_{21}+\frac{12 \mathrm{c}^{2}}{5} \grave{\mathbf{p}}_{30}
\end{aligned}
$$

lead to a circulant system of $2 n$ linear equations for computing $\mathbf{p}_{21}^{k}, \mathbf{p}_{12}^{k}$ since $\mathbf{p}_{21}^{k-1}=\grave{\mathbf{p}}_{12}, \mathbf{p}_{12}^{k-1}=\grave{\mathbf{p}}_{21}, \mathbf{p}_{21}^{k}=\grave{\mathbf{p}}_{21}, \mathbf{p}_{12}^{k}=$ $\dot{\mathbf{p}}_{12}$.

Similarly, the assignment in each local system of cyan crosses

$$
\begin{aligned}
\grave{\mathbf{p}}_{22} & :=\grave{\mathbf{p}}_{22}+\frac{(3 c-4)(3 c-5)}{5} \grave{\mathbf{p}}_{20}+(3 c-4) \grave{\mathbf{p}}_{21} \\
& -\frac{3 c(3 c-4)}{5} \grave{\mathbf{p}}_{30}-\frac{3 c}{2(c-2)}\left(\grave{\mathbf{p}}_{32}-\grave{\mathbf{p}}_{32}\right) \\
& -\frac{3 c^{2}}{4(c-2)(c-4)}\left(4\left(\grave{\mathbf{p}}_{42}-\grave{\mathbf{p}}_{42}\right)-c\left(\grave{\mathbf{p}}_{52}-\grave{\mathbf{p}}_{52}\right)\right)
\end{aligned}
$$

leads to a circulant system of $n$ linear equations for computing $\mathbf{p}_{22}^{k}$ since $\mathbf{p}_{22}^{k-1}=\grave{\mathbf{p}}_{22}, \mathbf{p}_{22}^{k}=\dot{\mathbf{p}}_{22}$.

(S3) The assignments of $\dot{\mathbf{p}}_{i j}, i>2$ (hollow boxes and hollow diamonds) can be made separate from other local systems, but interact across $\mathbf{q r}$ with systems of neighboring extraordinary nodes. The assignments guaranteeing smooth joints are

$$
\begin{aligned}
& \grave{\mathbf{p}}_{51}:=\grave{\mathbf{p}}_{50}+\frac{1}{4}\left(\grave{\mathbf{p}}_{52}-\dot{\mathbf{p}}_{52}\right) \\
& \dot{\mathbf{p}}_{51}:=\grave{\mathbf{p}}_{50}-\frac{1}{4}\left(\grave{\mathbf{p}}_{52}-\dot{\mathbf{p}}_{52}\right) \\
& \grave{\mathbf{p}}_{41}:=w_{0} \grave{\mathbf{p}}_{40}+\left(1-w_{0}\right) \grave{\mathbf{p}}_{50} \\
&+ w_{1}\left(\grave{\mathbf{p}}_{42}-\grave{\mathbf{p}}_{42}\right)+w_{2}\left(\grave{\mathbf{p}}_{52}-\dot{\mathbf{p}}_{52}\right) \\
& \dot{\mathbf{p}}_{41}:=w_{0} \grave{\mathbf{p}}_{40}+\left(1-w_{0}\right) \grave{\mathbf{p}}_{50}-w_{1}\left(\grave{\mathbf{p}}_{42}-\dot{\mathbf{p}}_{42}\right) \\
&- w_{2}\left(\grave{\mathbf{p}}_{52}-\grave{\mathbf{p}}_{52}\right) ; \\
& \grave{\mathbf{p}}_{31}:=\tilde{w}_{0} \grave{\mathbf{p}}_{30}+\left(1-\tilde{w}_{0}\right) \grave{\mathbf{p}}_{40}+\tilde{w}_{1}\left(\grave{\mathbf{p}}_{32}-\dot{\mathbf{p}}_{32}\right) \\
&+ \tilde{w}_{2}\left(\grave{\mathbf{p}}_{42}-\grave{\mathbf{p}}_{42}\right)+\tilde{w}_{3}\left(\grave{\mathbf{p}}_{52}-\grave{\mathbf{p}}_{52}\right) \\
& \dot{\mathbf{p}}_{31}:=\tilde{w}_{0} \grave{\mathbf{p}}_{30}+\left(1-\tilde{w}_{0}\right) \grave{\mathbf{p}}_{40}-\tilde{w}_{1}\left(\grave{\mathbf{p}}_{32}-\grave{\mathbf{p}}_{32}\right) \\
&-\tilde{w}_{2}\left(\grave{\mathbf{p}}_{42}-\dot{\mathbf{p}}_{42}\right)-\tilde{w}_{3}\left(\grave{\mathbf{p}}_{52}-\dot{\mathbf{p}}_{52}\right) \\
& w_{0}:=1-\frac{\mathrm{c}}{5}, w_{1}:=\frac{1}{4-\mathrm{c}}, w_{2}:=\frac{\mathrm{c}}{4(\mathrm{c}-4)} \\
& \tilde{w}_{0}:=1-\frac{2 \mathrm{c}}{5}, \tilde{w}_{1}:=\frac{1}{2(2-\mathrm{c})}, \\
& \tilde{w}_{2}:=\frac{\mathrm{c}}{(\mathrm{c}-2)(4-\mathrm{c})}, \tilde{w}_{3}:=\frac{1}{4(\mathrm{c}-2)(\mathrm{c}-4)}
\end{aligned}
$$

\subsection{Matching the tensor-border $\mathbf{b}$}

While the assignments (8) match the degree-raised tensorborder $\mathbf{b}$, the assignments (9) are incompatible with a $C^{2}$ prolongation of $\mathbf{b}$. (If second order Hermite data of $\mathbf{b}$ is to be 


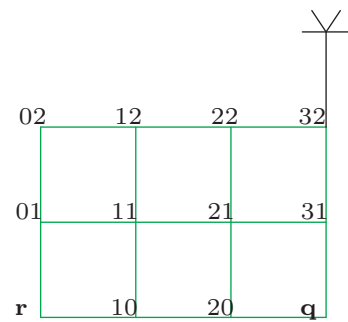

(a) initial $\mathbf{b}$

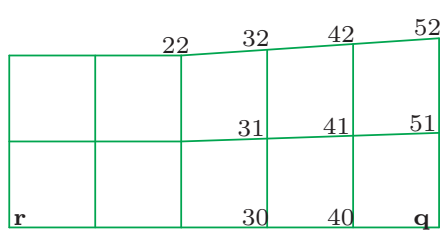

(b) adjusted tensor-border a
Figure 5: The indices of the (a) initial bi-3 tensor-border $\mathbf{b}$ and (b) its bi-5 adjustment $\mathbf{a}$. The boundary curves are degree-raised, hence remain geometrically unchanged.

preserved exactly, the reparameterization of the tensor-border results in higher degree as in [LS08] (bi-7) and [KP16] (bi-6)).

However for MSV meshes, due to their structural symmetry with respect to $\mathbf{r}$, o, within caps across qo and across rq between caps (cf. Fig. 3), we can adjust the initial bi-3 depth 2 tensor-borders $\mathbf{b}^{\alpha}$ to new bi-5 depth 2 tensor-borders $\mathbf{a}^{\alpha}$ that allow for a $G^{2}$ construction. In particular, at a meeting point $\mathbf{q}$ of four tensor-borders $\mathbf{a}^{\alpha}$ with coefficients $\mathbf{a}_{i j}^{\alpha}$ (see Fig. 6), the following observation is easily verified.

Observation. Consider four tensor-borders $\mathbf{a}^{\alpha}$ meeting at a point of type q. The coefficients are indexed by subscripts as in Fig. 5 (differing from Fig. 3). If the three layers $\mathbf{a}_{3 j}^{\alpha}, \mathbf{a}_{5 j}^{\alpha}$, $j \in\{0,1,2\}$ are $C^{2}$ connected then so are the $\mathbf{a}_{4 j}^{\alpha}$, defined by (8) and (9).

We set $\mathbf{a}_{i j}, i=0, \ldots, 5, j=0,1,2$ as follows. Degreeraise $\mathbf{b}$ to $\bar{b}$ of degree 5 and preserve the boundary by setting $\mathbf{a}_{i 0}=\overline{\mathbf{b}}_{i 0}$. For $j=1,2$ set

$$
\mathbf{a}_{3 j}:=\sum_{r=1}^{3} \sum_{s=0}^{2} \nu_{r s}^{3 j} \mathbf{b}_{r s}, \mathbf{a}_{5 j}:=\sum_{s=0}^{2} \nu_{3 s}^{5 j} \mathbf{b}_{3 s},
$$

where $\nu_{30}^{3 j}$ are chosen so that $\sum_{r=1}^{3} \sum_{s=0}^{2} \nu_{r s}^{3 j}=1$ and $\nu_{30}^{5 j}$ so that $\sum_{s=0}^{2} \nu_{3 s}^{5 j}=1$.

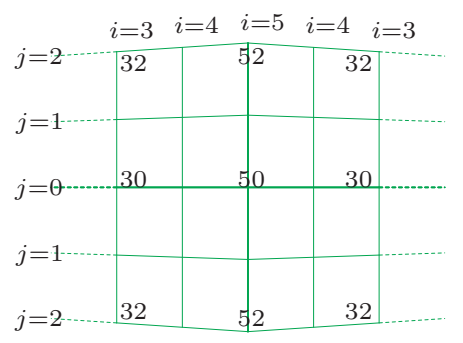

Figure 6: Indices $i j$ of adjusted tensor-borders $\mathbf{a}^{\alpha}$ with coefficients $\mathbf{a}_{i j}^{\alpha}$ meeting at a point of type $\mathbf{q}$ (cf. Fig. 3d).

Due to the symmetry of construction, it is easy to check that $\mathbf{a}_{3 j}, \mathbf{a}_{5 j}, j=0,1,2$, satisfy the assumptions of the Observation if we choose

$$
\begin{aligned}
\nu_{32}^{51} & :=0, \nu_{31}^{51}:=\frac{1}{2} \nu_{31}^{52}+\nu_{32}^{52} \\
\nu_{i 2}^{31} & :=0, \nu_{i 1}^{31}:=\frac{1}{2} \nu_{i 1}^{32}+\nu_{i 2}^{32}, i=1,2,3 \\
\nu_{20}^{31} & :=\frac{3}{5}-\nu_{21}^{31}, \nu_{10}^{31}:=\frac{3}{10}-\nu_{11}^{31} .
\end{aligned}
$$

\subsection{Explicit solution of the circulant systems}

The system (6) of $2 n$ equations has a unique solution unless $n=6$. In the latter case one unknown can be taken as free and we choose the BB-coefficient $\mathbf{p}_{21}^{0}$. In the system (7) of $n$ equations we choose, for any $n, \mathbf{p}_{22}^{0}$ as free and solve a subsystem of $n-1$ equations for $\mathbf{p}_{22}^{k}, k=1, \ldots, n-1$. For $n=5,6,7,8$ one can check that the remaining equation holds if

$$
\nu_{10}^{32}:=\tau_{n}-\frac{1}{2} \nu_{20}^{32}+\bar{\tau}_{n}\left(\nu_{21}^{32}+2 \nu_{22}^{32}+2 \nu_{11}^{32}+4 \nu_{12}^{32}\right),
$$

where with $\mathrm{c}:=\cos \frac{2 \pi}{n}$

\begin{tabular}{lll}
$n$ & $\tau_{n}$ & $\bar{\tau}_{n}$ \\
\hline 5 & $\frac{3(24-77 c)}{10(96 c-29)}$ & $\frac{16 c-5}{2(96 c-29)}$ \\
6 & $\frac{3}{20}$ & $-\frac{1}{14}$ \\
7 & $\frac{3\left(-553-1572 c+4096 c^{2}\right)}{40\left(160+471 c-1106 c^{2}\right)}$ & $\frac{28+79 c-210 c^{2}}{2\left(160+471 c-1106 c^{2}\right)}$ \\
8 & $\frac{3(1-8 c)}{20(c-4)}$ & $\frac{c}{2(c-4)}$
\end{tabular}

\subsection{The cap construction}

In order to obtain high surface quality, care must be taken when setting the remaining nine unconstrained parameters

$$
\mathcal{T}:=\left\{\nu_{31}^{32}, \nu_{32}^{32}, \nu_{20}^{32}, \nu_{21}^{32}, \nu_{22}^{32}, \nu_{11}^{32}, \nu_{12}^{32}, \nu_{31}^{52}, \nu_{32}^{52}\right\}
$$

Then the following algorithm can be invoked separately for each cap of $n$ patches.

\section{Algorithm bi-5}

Input: a local MSV mesh, consisting of the 2-link of one extraordinary node with $n$ neighbors (Fig. 3a,Fig. 7).

Output: a surface cap consisting of $n$ polynomial patches of degree bi-5. The cap is internally $G^{2}$ and is $C^{2}$ connected to its neighbor caps.

1. For $k=0, \ldots, n-1$ set the extraordinary point $\mathbf{p}_{00}=\mathbf{p}_{00}^{k}$ to the limit point of Catmull-Clark subdivision [HKD93]

$$
\begin{aligned}
\mathbf{p}_{00} & :=\frac{n}{n+5} \mathbf{c}^{7}+\sum_{i=0}^{n-1}\left(\gamma_{5} \mathbf{c}_{5}^{i}+\gamma_{6} \mathbf{c}_{6}^{i}\right), \\
\gamma_{5} & :=\frac{1}{n(n+5)}, \gamma_{6}:=\frac{4}{n(n+5)} .
\end{aligned}
$$

2. Set the tensor-border of $\mathbf{p}$ to $\mathbf{a}$, the adjusted tensor-border of the MSV mesh obtained by (10). 
3. Resolve all smoothness constraints by expressing all BBcoefficients in terms of a and the six coefficients $\mathcal{P}:=$ $\left\{\mathbf{p}_{i j}^{0}, \mathbf{p}_{22}^{0}\right\}, 1 \leq i+j \leq 2$, (plus $\mathbf{p}_{21}^{0}$ if $n=6$ ) according to $(5),(6),(7),(8),(9)$.

4. Determine $\mathcal{P}$ by minimizing the functional

$$
\mathcal{F}_{k} f:=\int_{0}^{1} \int_{0}^{1} \sum_{i+j=k, i, j \geq 0} \frac{k !}{i ! j !}\left(\partial_{s}^{i} \partial_{t}^{j} f(s, t)\right)^{2} d s d t
$$

over all bi-5 patches taking $\mathcal{F}_{4}$ for $n=5,6$ and $\mathcal{F}_{3}$ for $n=7,8$ as determined by experimentation.

Applying the algorithm with $\mathcal{T}$ still in symbolic representation to the characteristic configuration of Catmull-Clark subdivision [CC78] and then minimizing (combinations of) functionals $\mathcal{F}_{k}$ (and similar ones), a strategy that worked for general high-quality constructions [KP15b, KP16] failed to deliver $\mathcal{T}$ that result in good surfaces for test data. Only by diligent experimenting with the test data, did we find a good choice, namely setting to zero

$$
0=\nu_{30}^{41}=\nu_{30}^{42}=\nu_{30}^{31}=\nu_{30}^{32}=\nu_{31}^{32}=\nu_{20}^{32} .
$$

Applying the algorithm to the characteristic Catmull-Clark data and minimizing then determines $\nu_{31}^{52}, \nu_{22}^{32}$ and $\nu_{11}^{32}$.

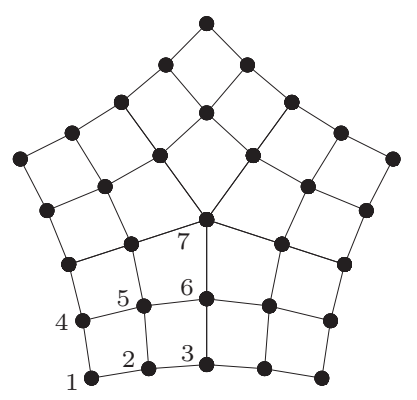

Figure 7: MSV mesh indices $m$ of $h_{i j}^{k, m}$. The points $\mathbf{c}_{m}^{k}$ form the 2-link of the central extraordinary node.

\subsection{Implementation via generating functions}

'Algorithm bi-5' works for each coordinate of the $G^{2}$ surface separately. When all input net points $\mathbf{c}_{m}^{k}$ have value 0 , except for $\mathbf{c}_{m}^{0}=1$ for one of the seven mesh points marked $\mathbf{o}, \mathbf{q}, \mathbf{r}$ in Fig. 3a and labeled $m=1,2, \ldots, 7$ in Fig. 7, we obtain the scalar-valued bi- 5 coefficients

$h_{i j}^{k, m} \in \mathbb{R}, \quad k=0, \ldots, n-1, m=1, \ldots, 7, i, j \in\{0, \ldots, 5\}$,

where the coefficients $h_{i j}^{0,7}=\ldots=h_{i j}^{n-1,7}$ correspond to $\mathbf{o}$. Then the BB-coefficients of the cap are

$$
\mathbf{p}_{i j}^{\kappa}:=h_{i j}^{0,7} \mathbf{c}_{7}^{0}+\sum_{k=0}^{n-1} \sum_{m=1}^{6} h_{i j}^{k, m} \mathbf{c}_{m}^{\kappa-k}
$$

The superscript of $\mathbf{c}_{m}^{\kappa-k}$ is interpreted modulo $n$.

\subsection{A bi-5 example}

We present only one bi-5 example since visually, by highlight analysis and under curvature shading, the bi-5 and the following bi-4 constructions for MSV meshes are nearly indistinguishable. The specific example Fig. 8c demonstrates the importance of choosing the functional $\mathcal{F}_{k}$ carefully in (4.) of the Algorithm: see Fig. 8d vs. Fig. 8e.

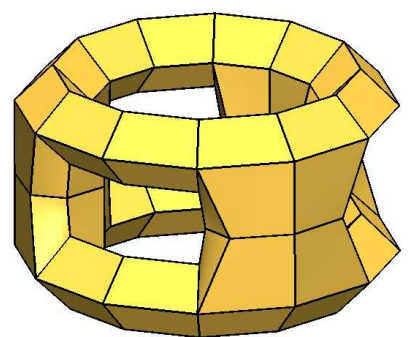

(a) $n=5 \mathrm{MSV}$ mesh

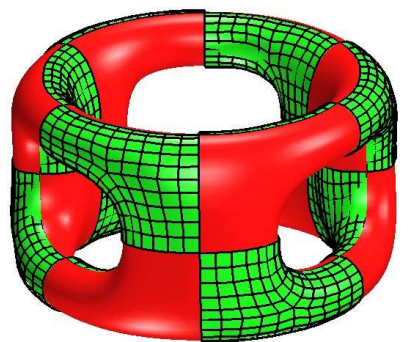

(b) 5-caps and BB-nets

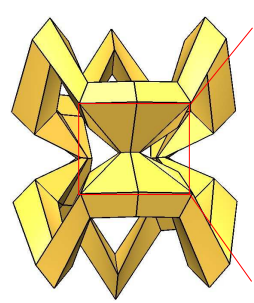

(c) $n=5 \mathrm{MSV}$ mesh

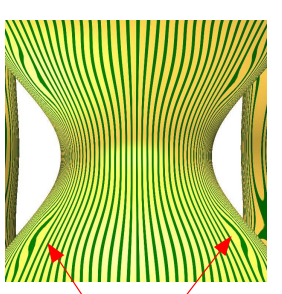

(d) $\mathcal{F}_{3}$

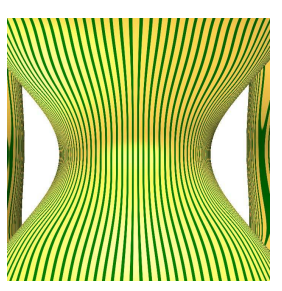

(e) $\mathcal{F}_{4}$
Figure 8: Bi-5 surfaces of genus 4 from MSV meshes with extraordinary nodes of valence 5 . (b) The connected BB-coefficients (BB-nets) of half the caps are shown. (c) is a deformation of (a). (d) red arrows point to highlight flaws when choosing $\mathcal{F}_{3}$.

\section{Bi-4 construction for MSV meshes}

The analogous construction of $G^{2}$ continuous surfaces of degree bi-4 requires a split into $2 \times 2$ bi-4 patches $\mathbf{p}^{s, k}, s \in$ $\{o, r, l, m\}$ as illustrated in Fig. 9 .

\subsection{Reparameterizing between sectors and solving constraints}

For the derivation of the $G^{2}$ constraints we abbreviate (see Fig. 9b)

$$
\grave{\mathbf{p}}:=\mathbf{p}^{o, k-1}, \grave{\mathbf{p}}:=\mathbf{p}^{o, k}, \underline{\grave{\mathbf{p}}}:=\mathbf{p}^{r, k-1}, \underline{\mathbf{p}}:=\mathbf{p}^{l, k}
$$

The reparameterizations (top) from o to the middle $\grave{\mathbf{p}}_{40}$, and from the middle to $\mathbf{q}$ (bottom) are

$$
\begin{array}{cl}
\text { top } \quad & a(u):=-1, b(u):=2 \mathrm{c}(1-u)+\mathrm{c} u, \\
& d(u):=0 \Rightarrow e(u):=b(u) b^{\prime}(u) \\
\text { bottom } & \underline{a}(u):=-1, \underline{b}(u):=\mathrm{c}(1-u) \\
& \underline{d}(u):=0 \Rightarrow \underline{e}(u):=\underline{b}(u) \underline{b}^{\prime}(u) .
\end{array}
$$

The $G^{1}$ and $G^{2}$ constraints (1) and (2) across oq amount to setting to zero four polynomials of degree 4 . This yields $n$ local systems between sector $k-1$ and $k$ for a total of 20 linear equations. Since we enforce internal $C^{2}$ continuity within each split 


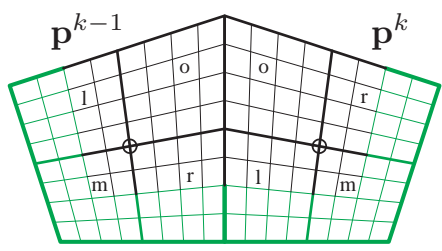

(a) rotationally-symmetric indexing

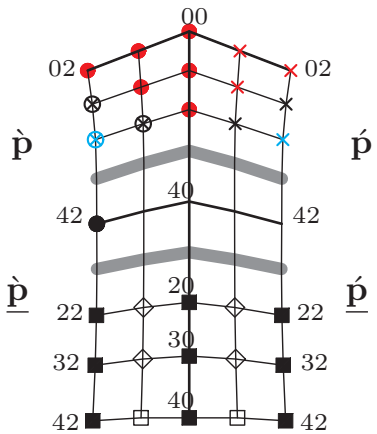

(b) sector-symmetric indexing
Figure 9: Green: adjusted tensor-border a. The extraordinary point has index 00 in both (a) and (b) and subscript 40 corresponds to q. The locally independent (free) BB-coefficients are marked by black and red disks, black and cyan circled crosses and the black squares. Thick-drawn grey layers are determined by adjacent layers and $C^{2}$ continuity.

sector, six BB-coefficients (thick grey layers in Fig. 9) are defined by the BB-coefficients in their adjacent layers. We present the algorithmic steps as similar as possible to the bi- 5 construction.

(S1) We account for the interactions between the $n$ local systems of equations at $\grave{\mathbf{p}}_{00}$ by selecting the six BB-coefficients $\mathbf{p}_{i j}^{o, 0}, 0 \leq i+j \leq 2$, that define a quadratic expansion of sector $k=0$ at extraordinary point and then express the corresponding (initially independent) BB-coefficients of the other sectors recursively via

$$
\begin{aligned}
\dot{\mathbf{p}}_{00}:= & \grave{\mathbf{p}}_{00}, \quad \dot{\mathbf{p}}_{10}:=\grave{\mathbf{p}}_{10}, \quad \dot{\mathbf{p}}_{20}:=\grave{\mathbf{p}}_{20} ; \\
\dot{\mathbf{p}}_{01}:= & -\grave{\mathbf{p}}_{01}+2 c \grave{\mathbf{p}}_{10}+2(1-\mathrm{c}) \grave{\mathbf{p}}_{00} ; \\
\dot{\mathbf{p}}_{11}:= & -\grave{\mathbf{p}}_{11}+\frac{3 \mathrm{c}}{2} \grave{\mathbf{p}}_{20}+\left(2-\frac{5 \mathrm{c}}{4}\right) \grave{\mathbf{p}}_{10}-\frac{\mathrm{c}}{4} \grave{\mathbf{p}}_{00} ; \\
\dot{\mathbf{p}}_{02}:= & \grave{\mathbf{p}}_{02}-\frac{16}{3} c \grave{\mathbf{p}}_{11}+4 \mathrm{c}^{2} \grave{\mathbf{p}}_{20}+\frac{4}{3}(4 c-3) \grave{\mathbf{p}}_{01} \\
& +\frac{2}{3} \mathrm{c}(14-13 \mathrm{c}) \grave{\mathbf{p}}_{10}+\frac{2}{3}\left(6-14 c+7 c^{2}\right) \grave{\mathbf{p}}_{00} .
\end{aligned}
$$

The last three assignments in (15) correspond to red crosses in Fig. 9b.

(S2) The assignments (black crosses)

$$
\begin{aligned}
\dot{\mathbf{p}}_{21} & :=-\grave{\mathbf{p}}_{21}+\left(2+\frac{\mathrm{c}}{2}\right) \grave{\mathbf{p}}_{20}-\frac{3 c}{4} \grave{\mathbf{p}}_{10}+\frac{c}{2} \underline{\mathbf{p}}_{20}-\frac{c}{4} \underline{\mathbf{p}}_{30} \\
\dot{\mathbf{p}}_{12} & :=\grave{\mathbf{p}}_{12}+\left(4-\frac{35 c}{6}-\frac{\mathrm{c}^{2}}{12}\right) \grave{\mathbf{p}}_{10}+\left(\frac{10 c}{3}-4\right) \grave{\mathbf{p}}_{11} \\
& +\frac{2 c}{3} \grave{\mathbf{p}}_{01}+\frac{\mathrm{c}(14-3 \mathrm{c})}{2} \grave{\mathbf{p}}_{20} \\
& -4 \mathrm{c} \grave{\mathbf{p}}_{21}+\frac{\mathrm{c}(13 \mathrm{c}-14)}{12} \grave{\mathbf{p}}_{00}+\mathrm{c}^{2} \underline{\mathbf{p}}_{20}-\frac{\mathrm{c}^{2}}{2} \underline{\mathbf{p}}_{30}
\end{aligned}
$$

lead to a circulant system of $2 n$ linear equations for computing $\mathbf{p}_{21}^{o, k}, \mathbf{p}_{12}^{o, k}$ since $\mathbf{p}_{21}^{o, k-1}=\grave{\mathbf{p}}_{12}, \mathbf{p}_{12}^{o, k-1}=\grave{\mathbf{p}}_{21}, \mathbf{p}_{21}^{o, k}=\dot{\mathbf{p}}_{21}$, $\mathbf{p}_{12}^{o, k}=\dot{\mathbf{p}}_{12}$. Similarly, since $\mathbf{p}_{22}^{o, k-1}=\grave{\mathbf{p}}_{22}, \mathbf{p}_{22}^{o, k}=\dot{\mathbf{p}}_{22}$, the assignment (cyan crosses)

$$
\begin{aligned}
\dot{\mathbf{p}}_{22} & :=\grave{\mathbf{p}}_{22}+2 \mathrm{c} \grave{\mathbf{p}}_{11}+\frac{\mathrm{c}(3 \mathrm{c}-14)}{4} \grave{\mathbf{p}}_{10}+\frac{\mathrm{c}^{2}}{4} \grave{\mathbf{p}}_{00} \\
& +\left(4+\frac{7 \mathrm{c}}{3}-\frac{7 \mathrm{c}^{2}}{6}\right) \grave{\mathbf{p}}_{20}-4\left(1+\frac{\mathrm{c})}{3}\right) \grave{\mathbf{p}}_{21} \\
& +\frac{\mathrm{c}(3+\mathrm{c})}{6}\left(2 \grave{\mathbf{p}}_{20}-\underline{\mathbf{p}}_{30}\right)+\frac{\mathrm{c}}{\mathrm{c}-3}\left(\underline{\mathbf{p}}_{22}-\underline{\mathbf{p}}_{22}\right) \\
& +\frac{\mathrm{c}(3+\mathrm{c})}{6(\mathrm{c}-3)(\mathrm{c}-6)}\left(6\left(\underline{\mathbf{p}}_{32}-\underline{\mathbf{p}}_{32}\right)-\mathrm{c}\left(\underline{\grave{\mathbf{p}}}_{42}-\underline{\mathbf{p}}_{42}\right)\right)
\end{aligned}
$$

in each local system leads to a circulant system of $n$ linear equations for computing $\mathbf{p}_{22}^{o, k}$.

$(\mathrm{S} 2 *)$ To retain the numbering of the bi-5 construction, the assignments for $\grave{\mathbf{p}}_{41}, \grave{\mathbf{p}}_{40}, \mathbf{p}_{41}, \mathbf{p}_{42}$ appear in Appendix A.

(S3) The assignments (hollow boxes and hollow diamonds in Fig. 9b)

$$
\begin{aligned}
\grave{\mathbf{p}}_{41}:= & \underline{\mathbf{p}}_{40}+\frac{1}{4}\left(\underline{\mathbf{p}}_{42}-\underline{\mathbf{p}}_{42}\right), \\
\dot{\mathbf{p}}_{41}:= & \underline{\grave{\mathbf{p}}}_{40}-\frac{1}{4}\left(\underline{\grave{\mathbf{p}}}_{42}-\underline{\mathbf{p}}_{42}\right) . \\
\underline{\mathbf{p}}_{31}:= & w_{0} \underline{\mathbf{p}}_{30}+\left(1-w_{0}\right) \grave{\mathbf{p}}_{40}+w_{1}\left(\underline{\mathbf{p}}_{32}-\underline{\mathbf{p}}_{32}\right) \\
& +w_{2}\left(\underline{\mathbf{p}}_{42}-\underline{\mathbf{p}}_{42}\right), \\
\underline{\mathbf{p}}_{31}:= & w_{0} \underline{\mathbf{p}}_{30}+\left(1-w_{0}\right) \underline{\mathbf{p}}_{40}-w_{1}\left(\underline{\mathbf{p}}_{32}-\underline{\mathbf{p}}_{32}\right) \\
& -w_{2}\left(\underline{\mathbf{p}}_{42}-\underline{\mathbf{p}}_{42}\right) ; \\
\underline{\mathbf{p}}_{21}:= & \tilde{w}_{0} \underline{\mathbf{p}}_{20}+\left(1-\tilde{w}_{0}\right) \grave{\mathbf{p}}_{30}+\tilde{w}_{1}\left(\underline{\mathbf{p}}_{22}-\underline{\mathbf{p}}_{22}\right) \\
& +\tilde{\tilde{w}}_{2}\left(\underline{\mathbf{p}}_{32}-\underline{\mathbf{p}}_{32}\right)+\tilde{w}_{3}\left(\underline{\mathbf{p}}_{42}-\underline{\mathbf{p}}_{42}\right), \\
\underline{\mathbf{p}}_{21}:= & \tilde{w}_{0} \underline{\mathbf{p}}_{20}+\left(1-\tilde{w}_{0}\right) \grave{\mathbf{p}}_{30}-\tilde{w}_{1}\left(\underline{\mathbf{p}}_{22}-\underline{\mathbf{p}}_{22}\right) \\
& -\tilde{w}_{2}\left(\underline{\mathbf{p}}_{32}-\underline{\mathbf{p}}_{32}\right)-\tilde{w}_{3}\left(\underline{\mathbf{p}}_{42}-\underline{\mathbf{p}}_{42}\right),
\end{aligned}
$$

where

$$
\begin{aligned}
& w_{0}:=1-\frac{c}{8}, w_{1}:=\frac{3}{2(6-\mathrm{c})}, w_{2}:=\frac{\mathrm{c}}{4(\mathrm{c}-6)} ; \\
& \tilde{w}_{0}:=1-\frac{\mathrm{c}}{4}, \tilde{w}_{1}:=\frac{3}{4(3-\mathrm{c})}, \\
& \tilde{w}_{2}:=\frac{3 \mathrm{c}}{2(\mathrm{c}-3)(6-\mathrm{c})}, \tilde{w}_{3}:=\frac{\mathrm{c}^{2}}{4(\mathrm{c}-3)(\mathrm{c}-6)}
\end{aligned}
$$

enforce $G^{2}$ continuity.

Analogous to the bi-5 construction, the circulant system (16) of $2 n$ equations has a unique solution unless $n=6$. In the latter case one unknown can be taken as free and we choose the BB-coefficient $\mathbf{p}_{21}^{o, 0}$. The system (17) of $n$ equations allows choosing $\mathbf{p}_{22}^{o, 0}$ as free and solving any $n-1$ equations for $\mathbf{p}_{22}^{o, k}, k=1, \ldots, n-1$ provided a careful choice is made in adjusting the tensor-border. Expressing, as in (10), the second-order expansion of $\mathbf{p}$ and $\mathbf{p}$ in terms of the tensor-border b and scalar weights $\nu_{r s}^{i j}$, the remaining equation holds for $n=5,6,7,8,9,10$ if the weights satisfy one constraint, (21) developed below. 


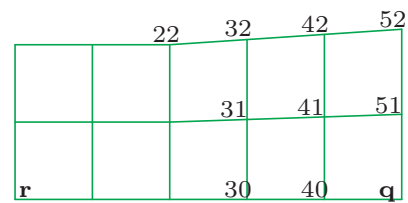

(a) Adjusted tensor-border a of a bi5 patch

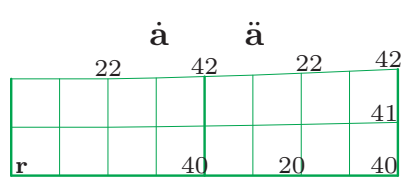

(b) Transformation to bi-4 form
Figure 10: The indices of (a) the adjusted tensor-border a of the bi-5 patch and (b) its transformation to two bi-4 pieces à, ä.

\subsection{Transformation of the adjusted tensor-border a to bi-4 form}

The adjusted tensor-border consists of two $C^{2}$-connected pieces $\dot{\mathbf{a}}$ and $\ddot{\mathrm{a}}$ of degree 4 and depth 2 (see Fig. 10b). While the adjustment at $\mathbf{q}$ is analogous to the bi-5 construction, the same strategy of setting parameters $\nu_{r s}^{i j}$ did not lead to quality surfaces. Instead, we make the bi-4 surfaces inherit the adjusted tensor-border from the bi-5 construction as follows.

(i) As in (10), for $j=1,2$ we set the BB-coefficients $\dot{\mathbf{a}}_{i j}\left(\ddot{\mathbf{a}}_{0 j}=\right.$ $\dot{\mathbf{a}}_{4 j}$ ) of degree 4 to

$$
\begin{aligned}
\ddot{\mathbf{a}}_{2 j} & :=\sum_{r=1}^{3} \sum_{s=0}^{2} \nu_{r s}^{2 j} \mathbf{b}_{r s}, \quad \ddot{\mathbf{a}}_{4 j}:=\sum_{s=0}^{2} \nu_{3 s}^{4 j} \mathbf{b}_{3 s} . \\
\nu_{32}^{41} & :=0, \nu_{31}^{41}:=\frac{1}{2} \nu_{31}^{42}+\nu_{32}^{42} ; \\
\nu_{r 2}^{21} & :=0, \nu_{r 1}^{21}:=\frac{1}{2} \nu_{r 1}^{22}+\nu_{r 2}^{22}, r=1,2,3 ; \\
\nu_{20}^{21} & :=\frac{1}{2}-\nu_{21}^{21}, \nu_{10}^{21}:=\frac{1}{2}-\nu_{11}^{21} .
\end{aligned}
$$

Then the layers $\ddot{\mathbf{a}}_{2 j}, \ddot{\mathbf{a}}_{4 j}, j=0,1,2$, together with the assignments of (S3) guarantee $C^{2}$ continuity at points of type $\mathbf{r}$ analogous to the Observation in the bi-5 construction. For $n=5,6,7,8,9,10$ the remaining equation of the system (17) holds if

$$
\nu_{10}^{22}:=\tau_{n}-\frac{1}{2} \nu_{20}^{22}+\bar{\tau}_{n}\left(\nu_{21}^{22}+2 \nu_{22}^{22}+2 \nu_{11}^{22}+4 \nu_{12}^{22}\right),
$$

where $\tau_{n}, \bar{\tau}_{n}$ are listed in Appendix B.

(ii) To determine the remaining free coefficients $\nu_{r s}^{2 j}, \nu_{3 s}^{4 j}$, we split the adjusted bi-5 tensor-border into halves, express the second-order expansion at $\mathbf{q}$ in bi-4 form in terms of the original border coefficients $\mathbf{b}_{i j}$ and compare this expansion to the expansion of $\ddot{\mathbf{a}}$ at $\mathbf{q}$ in terms of $\mathbf{b}_{i j}$. For valences $n=9,10$, we set the free $\nu_{r s}^{2 j}, \nu_{3 s}^{4 j}$ to

$$
\nu_{r s}^{i j, n}:=\nu_{r s}^{i j, 7}+\frac{\nu_{r s}^{i j, 8}-\nu_{r s}^{i j, 7}}{\mathrm{c}_{8}-\mathrm{c}_{7}}\left(\mathrm{c}_{n}-\mathrm{c}_{7}\right), \mathrm{c}_{n}:=\cos \frac{2 \pi}{n} .
$$

(iii) The bi-4 second-order BB-form coefficients $\dot{\mathbf{a}}_{i j}, i, j \in$ $\{0,1,2\}$ at $\mathbf{r}$ match the bi- 4 expansion of the split and adjusted tensor-border $\mathbf{b}$. Then we set

$$
\begin{array}{r}
\dot{a}_{4 j}:=-\frac{1}{4}\left(\dot{a}_{1 j}+\ddot{a}_{3 j}\right)+\frac{3}{4}\left(\dot{a}_{2 j}+\ddot{a}_{2 j}\right), \\
\ddot{a}_{0 j}:=\dot{a}_{4 j}, j=0,1,2,
\end{array}
$$

and fix the remaining BB coefficients $\dot{a}_{3 j}, \ddot{a}_{1 j}$ to join $C^{2}$ the pieces $\dot{a}$ and $\ddot{a}$ (One may check that $\dot{a}$ and $\ddot{a}$ are then $C^{3}$ connected). Their combined boundary is the boundary of $\mathbf{b}$, degree-raised and split into two pieces.

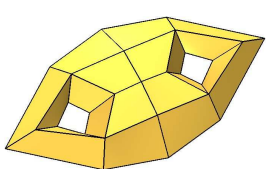

(a) MSV mesh, $n=5$

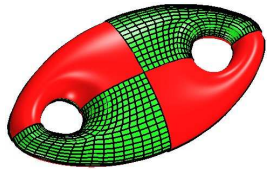

(b) 5-sided caps and their BB-nets

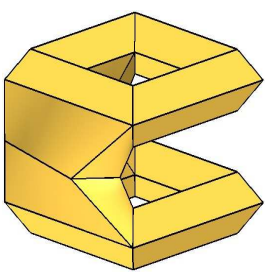

(d) MSV mesh, $n=5$

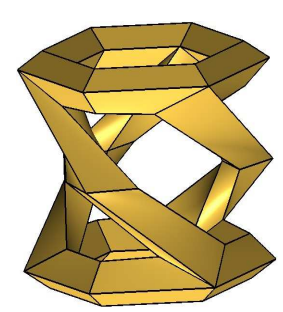

(g) MSV mesh, $n=6$

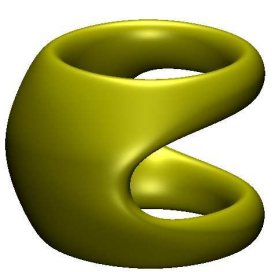

(e) scaffold surface

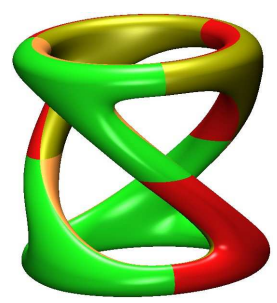

(h) 6-sided caps

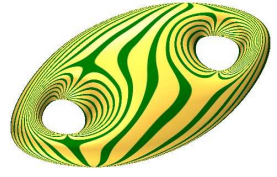

(c) highlight lines

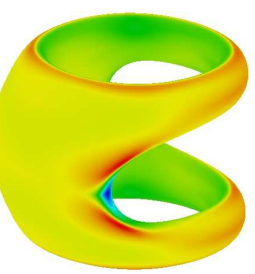

(f) Gauss curvature

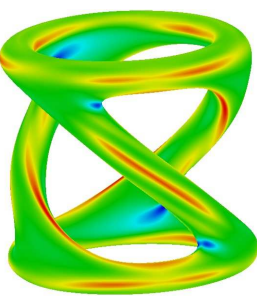

(i) mean curvature
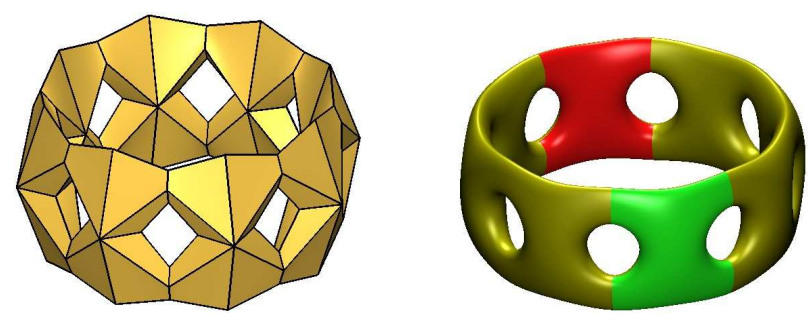

(j) $n=8$, genus 9
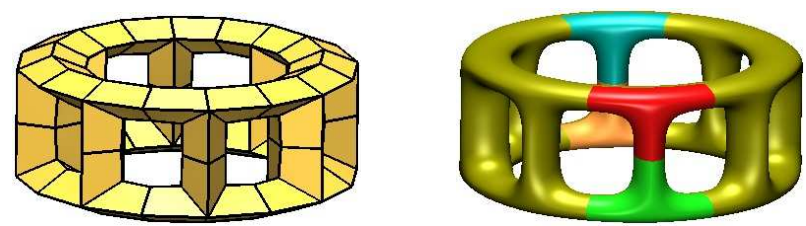

(k) $n=6$, genus 9
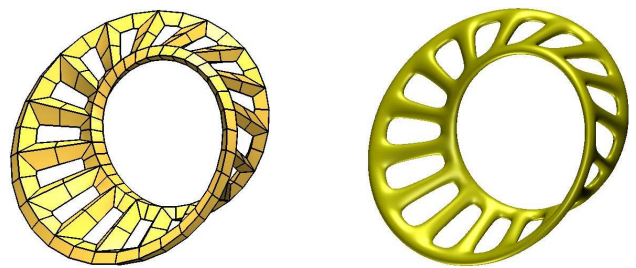

(1) $n=6$, genus 17

Figure 11: Design with MSV meshes. (top two rows) Valence 5 allows designing surfaces of genus 2 . $(\mathrm{g})$ is the sibling of Fig. 8a but using half the number of extraordinary nodes due to the different valence. 

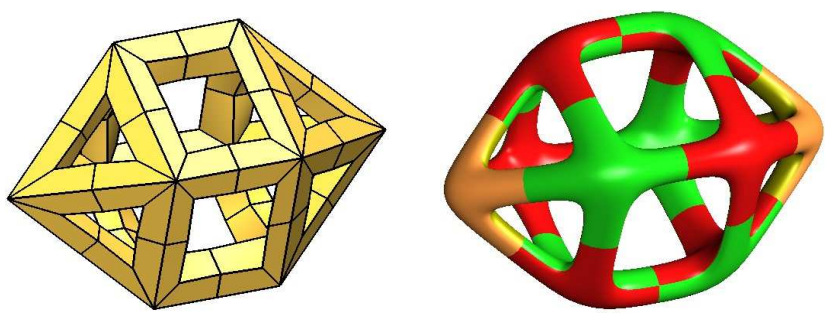

(a) Mesh and surface of genus 11 , extraordinary nodes of valence 8
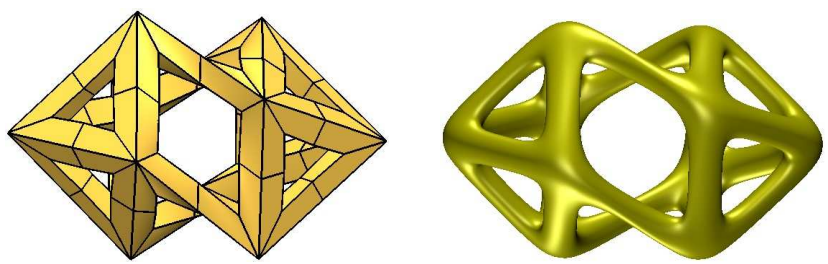

(b) Genus 11, valence 8
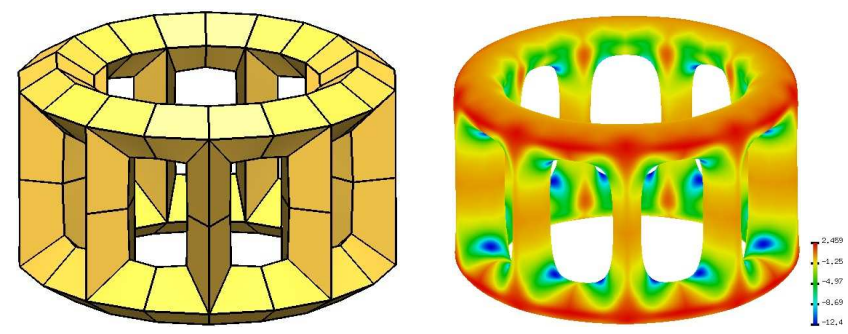

(c) Surface of genus 10, extraordinary nodes of valence 6
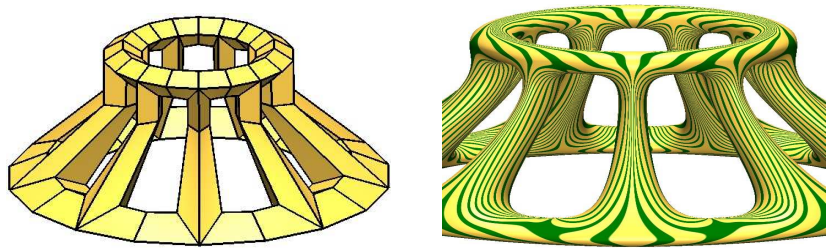

(d) Genus 10, valence 6

Figure 12: Bi-4 surfaces from MSV meshes. (left) MSV meshes. (right) scaffold surfaces with (a) colored neighborhoods, (c) Gauss curvature shading, (d) highlight lines.
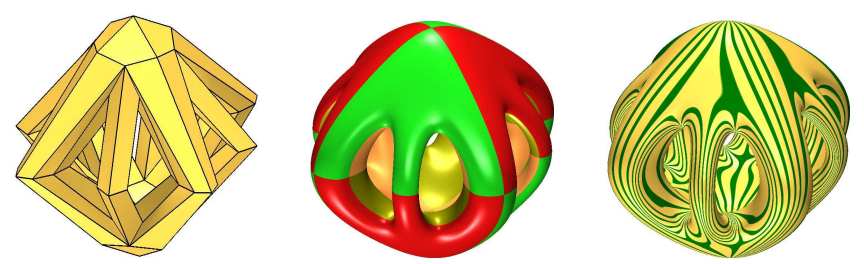

(a) Surface of genus 7 , extraordinary nodes of valence 7
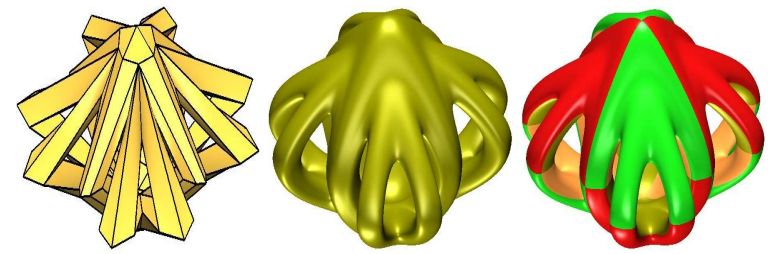

(b) genus 11 , valence 9

Figure 13: Bi-4 surfaces, esoteric valences.

\subsection{Algorithm and implementation}

\section{Algorithm bi-4}

Input: a local MSV mesh, consisting of the 2-link of one extraordinary node with $n$ neighbors (Fig. 3a,Fig. 7).

Output: a surface cap consisting of $4 n$ polynomial patches of degree bi-4. The cap is internally $G^{2}$ and is $C^{2}$ connected to its neighbor caps.

1. Set the extraordinary point $\mathbf{p}_{00}=\mathbf{p}_{00}^{o, k}$ for all $k$ to the limit point of Catmull-Clark subdivision.

2. Set the tensor-border of $\mathbf{p}$ to the adjusted pieces $\dot{\mathbf{a}}, \mathbf{a}$ of (20).

3. By $\mathcal{P}:=\left\{\mathbf{p}_{i j}^{o, 0}, \mathbf{p}_{22}^{o, 0}, \mathbf{p}_{24}^{o, k}, \mathbf{p}_{44}^{o, k}\right\}, 1 \leq i+j \leq 2, k=$ $0, \ldots, n-1$, denote the set of $6+2 n$ unconstrained BBcoefficients plus $\mathbf{p}_{21}^{o, 0}$ if $n=6 .\left(\mathbf{p}_{24}^{o, k-1}=\grave{\mathbf{p}}_{42}\right.$; and $\mathbf{p}_{44}^{o, k}$ is the point shared by all four pieces of the $2 \times 2$ split). Express all remaining BB-coefficients in terms of $\mathcal{P}$ and $\dot{\mathbf{a}}, \ddot{a}$ to resolve all smoothness constraints including the interior $C^{2}$ join of the $2 \times 2$-split patches.

4. Determine $\mathcal{P}$ by minimizing, over all bi-4 patches, the functional $\mathcal{F}_{4}$ for $n=5,6$ and $\mathcal{F}_{3}$ for $n=7,8,9,10$.

The implementation is analogous to that of the bi-5 construction.

\subsection{Bi-4 examples}

Although the connectivity of MSV meshes is severely restricted they yield a range of surfaces of genus greater than one that have good highlight lines thanks to the carefully set parameters. Fig. 11 illustrates this claim for valence $n=5$ and genus 2 and valence $n=6$ and genus 4 surfaces. Subsequent examples and Fig. 12 make the point that, even though structurally constrained, MSV meshes allow for a range of freeform shapes. Often higher valence can be replaced by a more complex arrangement of lower $n$ neighborhoods for increased flexibility. Fig. 11k was initially modeled using valence 8 (cf. Fig. 11j) then redesigned with twice the number of (valence 6) neighborhoods. The 'platonic' scaffold surfaces of Fig. 19 naturally include valence $n=10$ and shows that high valences can be dealt with by the Algorithm (as also illustrated by the esoteric valences and H.R. Giger-style designs of Fig. 13).

\section{Sphere-like surfaces}

So far, we focused on the most common valencies $n=$ $5,6,7,8,9,10$ since, for the bulk of scaffold surfaces no other valencies are required. Valence $n=3$ does not seem relevant for scaffold surfaces. MSV meshes with nodes of valence 3 model a once-refined cube, hence genus 0 sphere-like surfaces. Since, anyhow, the earlier construction does not directly transfer to the case $n=3$, we prefer to present a separate new class of free-form surfaces based on meshes with polar structure. The resulting sphere-like surfaces do not require minimality, yet are curvature continuous and of degree only bi-4. 


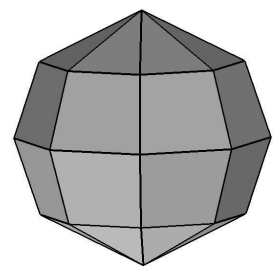

(a) $n=8$ pole

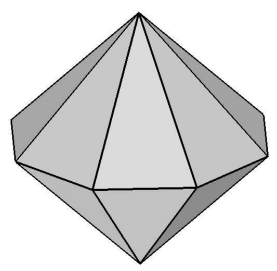

(b) minimal polar mesh

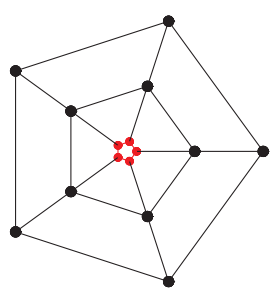

(c) combinatorial 2-link

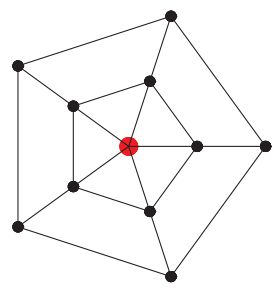

(d) geometric 2-link

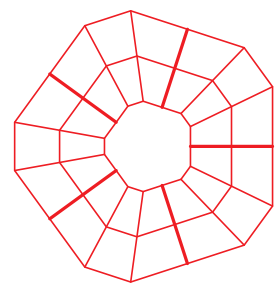

(e) tensor-border

Figure 14: Polar structure. Polar meshes with (a) three parallels, (b) one parallel. (c) combinatorial quad structure; (d) geometric structure with triangular faces; (e) Polar tensor-border b of degree 3 and depth 2.

Polar meshes consist of meridians through the pole(s) and periodic (latitudinal) parallels, see Fig. 14a. Except for the poles, all nodes have valence 4 , making them single-valence but not necessarily minimal. Minimality would require that poles are separated by exactly one parallel as in Fig. 14b. It is convenient to treat the polar meshes combinatorially as quad meshes (see Fig. 14c) with, at the irregularity, one edge collapsed to form the pole (Fig. 14d). Interpreting the quad mesh as a bicubic B-spline control net, and converting to BB-form yields a polar tensor-border $\mathbf{b}$ of degree 3 and depth 2 that serves as Hermite data for constructing the cap (Fig. 14d).

We construct polar surfaces whose patches join $C^{2}$ across parallels and whose parallels join $G^{2}$. We recall that two curve segments $f:[0,1] \rightarrow \mathbb{R}$ and $g:[0,1] \rightarrow \mathbb{R}$ join $G^{1}$ at a common point $f(1)=g(0)$ if for some scalar $\beta>0$ $g^{\prime}(0)=\beta f^{\prime}(1)$ and $G^{2}$ if additionally there exists $\gamma \in \mathbb{R}$ so that $g^{\prime \prime}(0)=\beta^{2} f^{\prime \prime}(1)+\gamma f^{\prime}(1)$.

The polar capping surfaces are based on a polar parameterization $\rho$ of the domain. The map $\rho$ is quadratic in the $u$ direction along parallels and linear in meridian $v$-direction (see Fig. 15a). The first sector $\rho_{1}$ of $\rho$ is defined as

$$
\begin{aligned}
& \rho_{1}:=v \mathbf{r}(u), \quad \mathbf{r}(u):=\sum_{i=0}^{2} \mathbf{r}_{i} B_{i}^{2}(u), \quad \alpha:=\frac{2 \pi}{n} \\
& \mathbf{r}_{0}:=(1,0), \mathbf{r}_{1}:=\left(1, \tan \frac{\alpha}{2}\right), \mathbf{r}_{2}:=(\cos \alpha, \sin \alpha) .
\end{aligned}
$$

The other sectors are copies of $\rho_{1}$ rotated by multiples of $\alpha$. By construction, $\rho$ is $C^{1}$, i.e. $\beta=1$, and, due to symmetry, $G^{2}$ with $\gamma:=2(\cos \alpha-1)$.

The map $\rho$ can be made more flexible by splitting $\mathbf{r}(u)$ as illustrated in Fig. 15b,c. Splitting then yields new continuity parameters $\beta_{i}, \gamma_{i}$ between the pieces. When the ratio between two pieces is $e: d$ then at the new splitting point $\beta^{\text {new }}:=$ $\frac{d}{e}, \gamma^{\text {new }}:=0$ (see Fig. 15c,top) and, at the original junction $\beta^{\text {new }}:=\frac{d}{e} \beta^{\text {old }}, \gamma^{\text {new }}:=\frac{d^{2}}{e} \gamma^{\text {old }}$ (Fig. 15c,bottom).

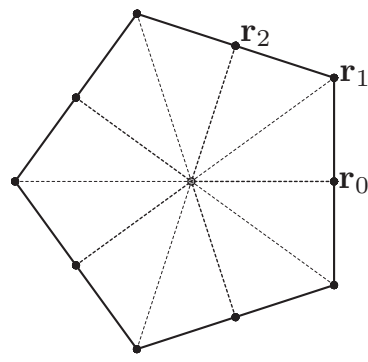

(a) $\rho$

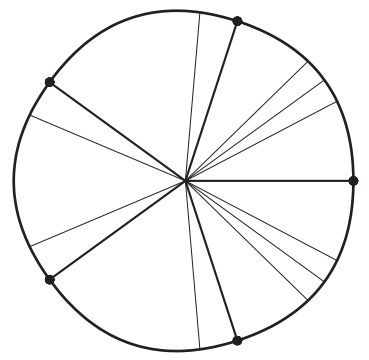

(b) $\rho$ partitioned

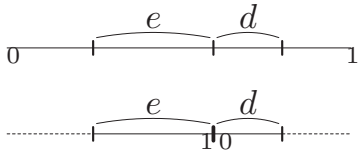

(c)

Figure 15: Polar parameterization (a) uniform $G^{2}$ piecewise quadratic construction in the periodic direction and linear in the meridian direction. (b) splitting uniform sectors into multiple pieces. (c) Two cases for calculation of $\beta$ and $\gamma$ from the ratio $e: d$ after a splitting point has been added. top: at new point (where intervals $e$ and $d$ meet) bottom: at the original junction point.

The steps of the construction of polar caps are as follows.

\section{Algorithm polar bi-4}

Input: a polar mesh, consisting of the 2-link of one extraordinary node with $n$ neighbors (Fig. 14d).

Output: a surface cap consisting of $n$ polynomial patches of degree bi-4. The cap is singular at the star point, internally $G^{2}$ and $C^{2}$ connected across parallels.

(P1) Express the composition $\mathbf{q} \circ \rho$ of $\rho$ with $\mathbf{q}$, a (still undefined) quadratic expansion at the origin $(0,0)$, in BBform of degree 4 along parallels and 3 along meridians (see Fig. 16a).

(P2) Fix the six coefficients of $\mathbf{q}$ per coordinate by minimizing the sum of distances between corresponding control points of the composition that are circled in Fig. 16a and the control points of the tensor-border $\mathbf{b}$ indicated as circles in Fig. 16b.

(P3) Interpolate the red and blue jets matched by patches of degree 5 along the meridians (and degree 4 along the parallels). This yields patches of degree 4,5 with one edge collapsed to form the pole.

(P4) Decrease the degree along the meridian from 5 (BBcoefficients shown in Fig. 16d, top), by splitting along the $v$-direction into either

- two $C^{3}$-connected pieces of degree 4 (Fig. 16e, middle); or

- into three $C^{2}$-connected pieces of degree 3 (Fig. 16e, bottom).

The bi-44 and bi-43 caps are nearly indistinguishable visually, via highlights or under curvature shading. The patches constructed in (P3) are $C^{2}$ connected to the input tensor-border 


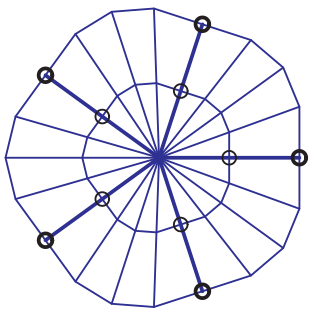

(a) $\mathbf{q} \circ \rho$

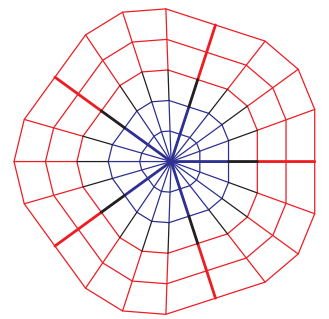

(c) cap of degree 4,5

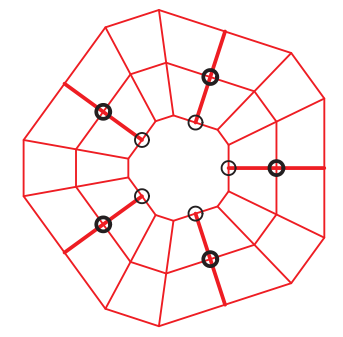

(b) tensor-border $\mathbf{b}$

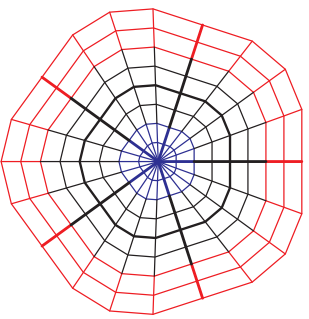

(d) cap of degree bi-4
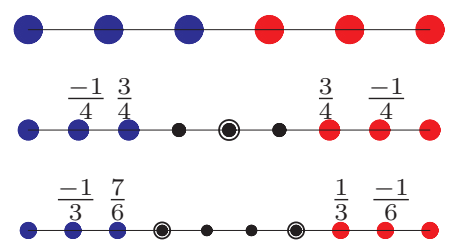

(e) masks for meridian degree reduction

Figure 16: Polar cap. (e) (middle) The degree 5 layer is split into two. The red and blue expansions are expressed in BB-form of degree 4 . The mask defines the circled common point of degree 4 pieces (cf. Section 4.2(iii) ). (bottom) The degree 5 layer is split into three. The red and blue expansions are expressed in BB-form of degree 3. The mask defines the left circled common point of the degree 3 pieces (the right circled point is defined by symmetry).

b and $G^{2}$ connected in the periodic direction of parallels and, as a composition of a $G^{2}$ and a $C^{\infty}$ map, they join with curvature continuity at a pole. Step ( $\mathrm{P} 4)$ does not change the curvature continuity established in (P3).

Comparison. Unlike minimal scaffold surfaces for valence $n=3$, polar surfaces do not need to be minimal to attain the low degree bi-4. Compared to [KP09], geometric continuity, specifically replacing a cubic $C^{2} \mathbf{r}$ by a quadratic $G^{2} \mathbf{r}$, allows for lower degree of the parallels (4 vs 6 ) - while the partitioning as in Fig. 15b provides flexibility for feature design. The lower degree comes at a cost: all parallels need to share the same $G^{2}$ reparameterization. However, for sphere-like surfaces this is not a major concern: it neither adds to the complexity of the construction nor stifles design (see Fig. 17f,g). The rational bi3 polar $G^{2}$ caps of [KP11] can model exact sphere caps, but generically have poorer shape.

\section{Conclusion}

We introduced a special class of curvature continuous surfaces of low and likely least polynomial degree and few(est) number of pieces for the special class of MSV meshes. MSV meshes occur in nature and engineering practice alike. We fo-

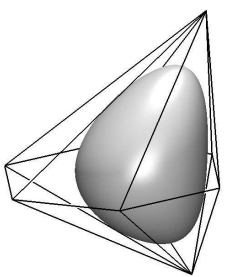

(a) $n=6$ polar MSV mesh

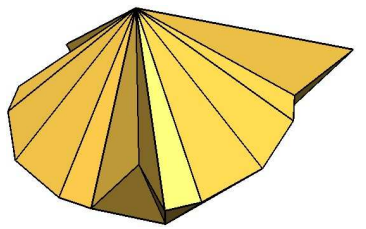

(d) polar MSV mesh

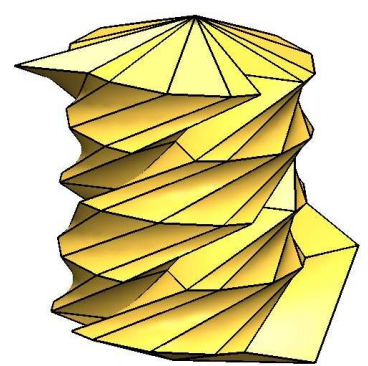

(f) polar drill bit (with cap (d))
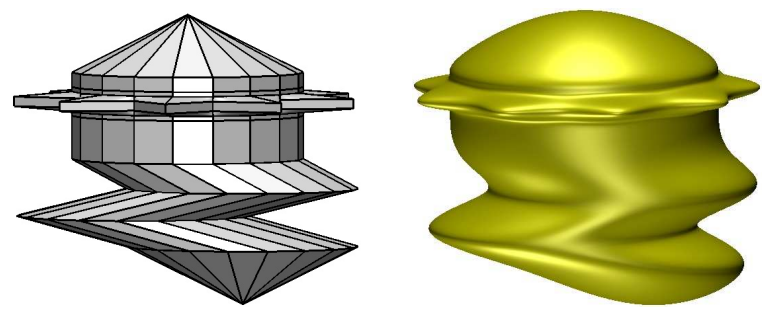

(g) polar art

Figure 17: $G^{2}$ polar surfaces of degree bi-4, genus 0 . Top row: (b) shows the BB-coefficients and (c) highlight shading looking down onto the pole.

cused on the main cases, MSV meshes with valence 5,6 or 8 but also covered more esoteric valences. Valence 3 requires special consideration. Since the result would be sphere-like surfaces, we opted to instead use a more flexible polar construction. Our curvature continuous polar surfaces of degree bi-4 have a simple derivation. Their key structure is the quadratic expansion at the pole. Together, the two families offer a structurally simple solution to the modeling of a common but special class of shapes. Curvature and highlight shading show the bi-4 scaffold surfaces to have shape at least as good as the state-ofthe-art methods of Table 1 and typically indistinguishable from [KP16].

[CC78] E. Catmull and J. Clark. Recursively generated B-spline surfaces on arbitrary topological meshes. Computer-Aided Design, 10:350-355, 1978. 
[GH89] John A. Gregory and Jorg M. Hahn. A C ${ }^{2}$ polygonal surface patch. Comp Aided Geom Design, 6(1):69-75, 1989.

[GZ99] John A. Gregory and Jianwei Zhou. Irregular $C^{2}$ surface construction using bi-polynomial rectangular patches. Comp Aided Geom Design, 16(5):423-435, 1999.

[HKD93] Mark Halstead, Michael Kass, and Tony DeRose. Efficient, fair interpolation using Catmull-Clark surfaces. In Proc 20th Annual Conference on Computer Graphics and Interactive Techniques, SIGGRAPH '93, pages 35-44, New York, NY, USA, 1993. ACM.

[Kic13] Przemyslaw Kiciak. Spline surfaces of arbitrary topology with continuous curvature and optimized shape. Computer-Aided Design, 45(2):154-167, 2013.

[KP] Kȩstutis Karčiauskas and Jörg Peters. Quad-net obstacle course. http://www.cise.ufl.edu/research/ SurfLab/shape_gallery.shtml. Accessed: 2015-05-05.

[KP09] K. Karčiauskas and J. Peters. Finite curvature continuous polar patchworks. In E. Hancock, R.R. Martin, and M. Sabin, editors, IMA Mathematics of Surfaces XIII Conference, pages 222-234, 2009.

[KP11] K. Karčiauskas and J. Peters. Rational $G^{2}$ splines. Graphical Models, 23(5):286-295, 2011.

[KP15a] Kȩstutis Karčiauskas and Jörg Peters. Biquintic $G^{2}$ surfaces via functionals. Computer Aided Geometric Design, pages 17-29, 2015.

[KP15b] Kȩstutis Karčiauskas and Jörg Peters. Improved shape for multisurface blends. Graphical Models, 8:87-98, 22015.

[KP16] Kȩstutis Karčiauskas and Jörg Peters. Minimal bi-6 $G^{2}$ completion of bicubic spline surfaces. Computer Aided Geometric Design, 41:10-22, Jan 2016.

[LS08] Charles T. Loop and Scott Schaefer. $G^{2}$ tensor product splines over extraordinary vertices. Comput. Graph. Forum, 27(5):1373-1382, 2008.

[Pet02a] J. Peters. $C^{2}$ free-form surfaces of degree (3,5). Computer-Aided Geometric Design, 19(2):113-126, 2002.

[Pet02b] J. Peters. Geometric continuity. In Handbook of Computer Aided Geometric Design, pages 193-229. Elsevier, 2002.

[Pra97] H. Prautzsch. Freeform splines. Comput. Aided Geom. Design, 14(3):201-206, 1997.

[Rei98] U. Reif. TURBS-topologically unrestricted rational $B$-splines. Constr. Approx., 14(1):57-77, 1998.

[Ye97] Xiuzi Ye. Curvature continuous interpolation of curve meshes. Computer Aided Geometric Design, 14(2):169-190, 1997.

\section{Appendix A}

Here and in Appendix B, $c:=\cos (2 \pi / n)$.

$$
\begin{aligned}
\grave{\mathbf{p}}_{40}:= & \frac{1}{4}\left(3 \grave{\mathbf{p}}_{20}-\grave{\mathbf{p}}_{10}+3 \underline{\mathbf{p}}_{20}-\underline{\mathbf{p}}_{30}\right) \\
\grave{\mathbf{p}}_{41}:= & \frac{3}{4} \grave{\mathbf{p}}_{21}-\frac{1}{4} \grave{\mathbf{p}}_{11}-\frac{\mathrm{c}}{32}\left(\grave{\mathbf{p}}_{00}-4 \grave{\mathbf{p}}_{10}+4 \grave{\mathbf{p}}_{20}\right)+\frac{12-\mathrm{c}}{16} \underline{\grave{\mathbf{p}}}_{20} \\
& +\frac{3 \mathrm{c}-8}{32} \grave{\grave{\mathbf{p}}}_{30}+\frac{9\left(\underline{\mathbf{p}}_{22}-\underline{\mathbf{p}}_{22}\right)}{16(3-\mathrm{c})} \\
& -\frac{(3+2 \mathrm{c})\left(6\left(\underline{\mathbf{p}}_{32}-\underline{\mathbf{p}}_{32}\right)-\mathrm{c}\left(\grave{\mathbf{p}}_{42}-\underline{\mathbf{p}}_{42}\right)\right)}{16(3-\mathrm{c})(6-\mathrm{c})} \\
\dot{\mathbf{p}}_{42}:= & \grave{\mathbf{p}}_{42}+\frac{\mathrm{c}}{8} \grave{\mathbf{p}}_{00}+\grave{\mathbf{p}}_{11}+\frac{\mathrm{c}^{2}-2 \mathrm{c}-4}{4} \grave{\mathbf{p}}_{10}+\left(\frac{2 \mathrm{c}}{3}-3\right) \grave{\mathbf{p}}_{21} \\
& +\frac{18-4 \mathrm{c}-\mathrm{c}^{2}}{6} \grave{\mathbf{p}}_{20}+\frac{\mathrm{c}(9-2 \mathrm{c})\left(2 \grave{\mathbf{p}}_{20}-\underline{\mathbf{p}}_{30}\right)}{24} \\
& +\frac{(9+2 \mathrm{c})\left(\underline{\mathbf{p}}_{22}-\underline{\mathbf{p}}_{22}\right)}{4(\mathrm{c}-3)} \\
& +\frac{\left(9+6 \mathrm{c}+2 \mathrm{c}^{2}\right)\left(6\left(\grave{\mathbf{p}}_{32}-\underline{\mathbf{p}}_{32}\right)-\mathrm{c}\left(\underline{\mathbf{p}}_{42}-\underline{\mathbf{p}}_{42}\right)\right)}{12(3-\mathrm{c})(6-\mathrm{c})} .
\end{aligned}
$$

The formula for $\dot{\mathbf{p}}_{41}$ is obtained from that for $\grave{\mathbf{p}}_{41}$ by exchanging, for $j=1,2, \grave{\mathbf{p}}_{i j} \leftrightarrow \dot{\mathbf{p}}_{i j}$ and $\underline{\mathbf{p}}_{i j} \leftrightarrow \underline{\mathbf{p}}_{i j}$.

\section{Appendix B}

The weights $\tau$ in the computation of $\nu_{10}^{22}$ are as follows.

\begin{tabular}{lll}
$n$ & $\tau_{n}$ & $\bar{\tau}_{n}$ \\
\hline 5 & $\frac{89-299 c}{16(41-136 c)}$ & $\frac{44 c-13}{4(41-136 c)}$ \\
6 & $\frac{7}{32}$ & $-\frac{7}{44}$ \\
7 & $\frac{1935+5800 c-12872 c^{2}}{64\left(226+667 c-1554 c^{2}\right)}$ & $\frac{-71-215 c+462 c^{2}}{4\left(226+667 c-1554 c^{2}\right)}$ \\
8 & $\frac{35+6 c}{32(6-c)}$ & $\frac{c+3}{4(c-6)}$ \\
9 & $\frac{797-5170 c+3332 c^{2}}{64\left(86-577 c+470 c^{2}\right)}$ & $-\frac{31-197 c+106 c^{2}}{4\left(86-577 c+470 c^{2}\right)}$ \\
10 & $\frac{24677+79880 c}{32(3239+10486 c)}$ & $\frac{-2377-7694 c}{4(3239+10486 c)}$ \\
\hline
\end{tabular}

\section{Acknowledgments.}

The work was supported in part by NSF Grant CCF1117695 .
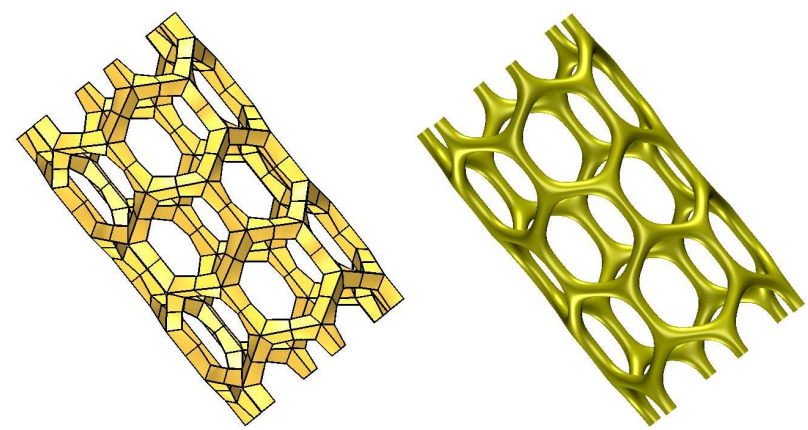

(a) MSV mesh and curvature continuous surface

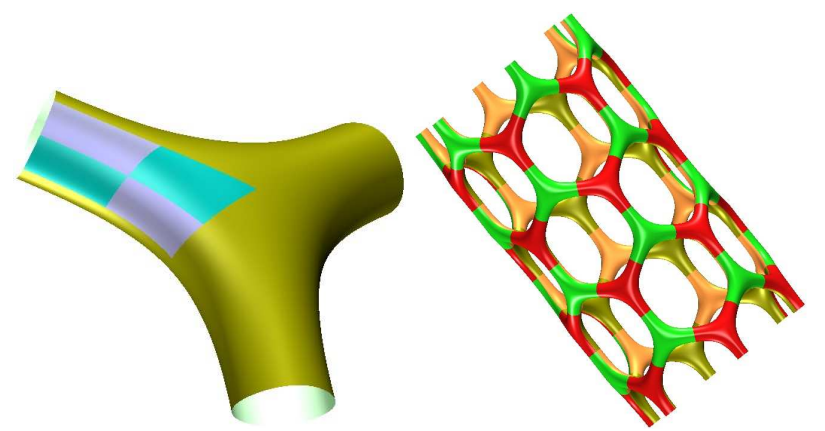

(b) structure (local $2 \times 2$ split, $n=6$ neighborhoods)

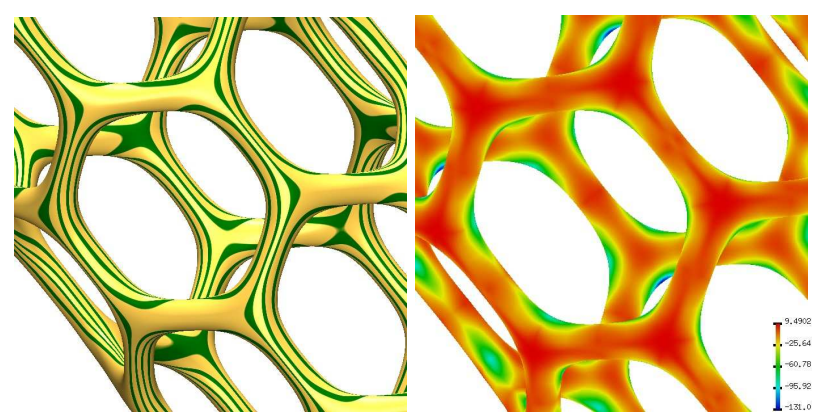

(c) shape (highlight lines, Gauss curvature)

Figure 18: Carbon nano structure as MSV mesh and scaffold surface. 

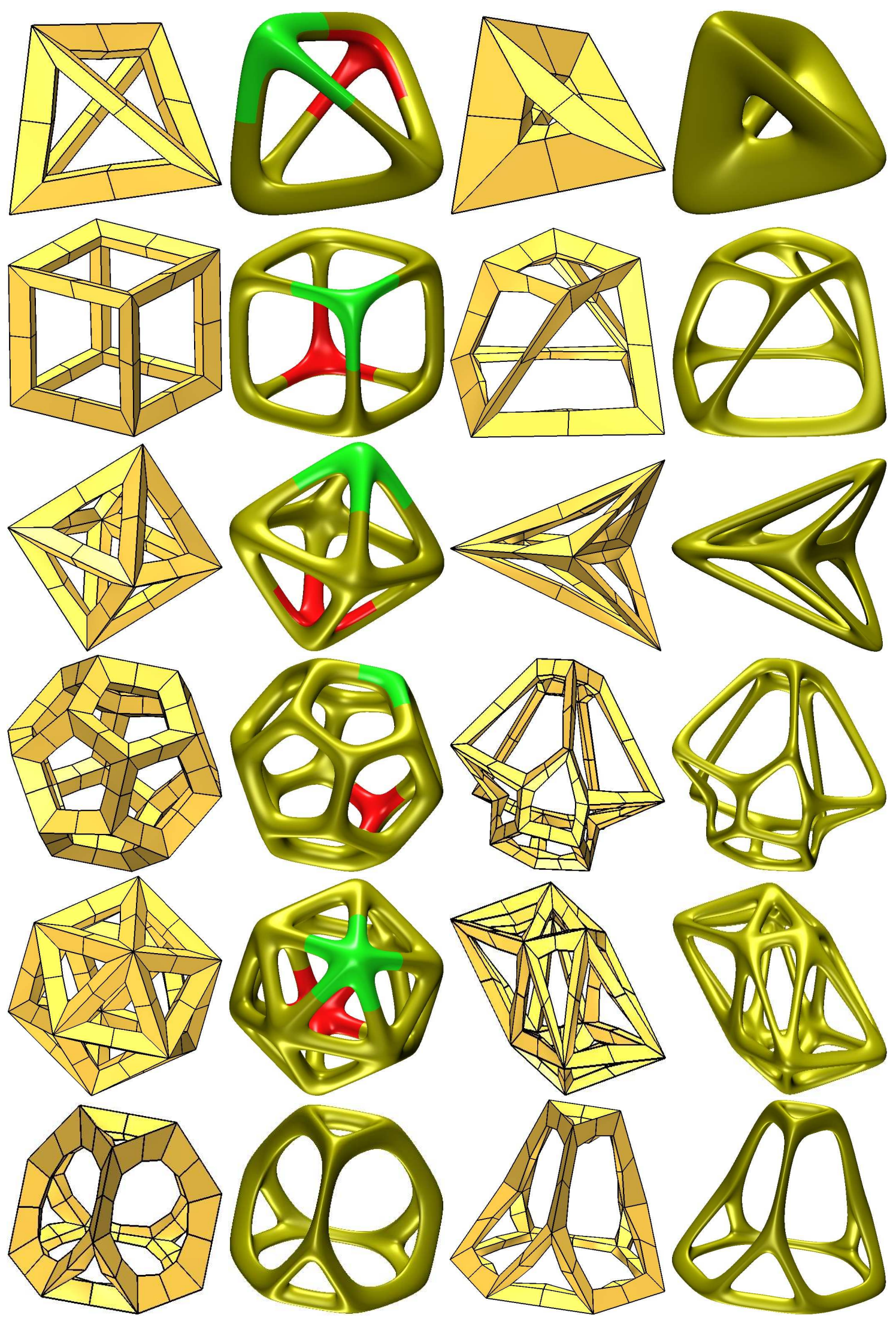

Figure 19: (row 1-5) Platonic MSV meshes and bi-4 scaffold surfaces with $n=6,6,8,6,10$ from top to bottom. (row 6 ) chopped tetrahedron, $n=6$. 